\title{
Enhanced Phase Mixing of Torsional Alfvén Waves in Stratified and Divergent Solar Coronal Structures, Paper I: Linear Solutions
}

\author{
C. Boocock ${ }^{1 \star}$ (D) and D. Tsiklauri ${ }^{2}$ (ID) \\ ${ }^{1}$ Queen Mary University of London, Mile End Road, London E1 4NS, UK, \\ ${ }^{2}$ School of Science and Technology, University of Georgia, 77a Kostava Street, 0171 Tbilisi, Georgia
}

Accepted 2021 November 26. Received 2021 November 25; in original form 2021 October 20

\begin{abstract}
We derive a corrected analytical solution for the propagation and enhanced phase mixing of torsional Alfvén waves, in a potential magnetic field with exponentially divergent field lines, embedded in a stratified solar corona. Further we develop a code named TAWAS which calculates the analytic solution describing torsional Alfvén waves using IDL software language. We then use TAWAS to demonstrate that both our correction to the analytic solution and the inclusion of wave reflection have a significant impact on Alfvén wave damping. We continue to utilise TAWAS by performing a parameter study in order to identify the conditions under which enhanced phase mixing is strongest. We find that phase mixing is the strongest for high frequency Alfvén waves in magnetic fields with highly divergent field lines and without density stratification. We then present a finite difference solver, Wigglewave, which solves the linearised evolution equations for the system directly. Comparing solutions from TAWAS and Wigglewave we see that our analytical solution is accurate within the limits of the WKB approximation but under-reports the wave damping, caused by enhanced phase mixing, beyond the WKB limit. Both TAWAS and Wigglewave solve the linearised governing equations and not the complete nonlinear MHD equations. Paper II will consider simulations that solve the full MHD equations including important nonlinear effects.
\end{abstract}

Key words: (magnetohydrodynamics) MHD - Plasmas - Waves - Sun: corona - Sun: oscillations

\section{INTRODUCTION}

In this paper we will consider the propagation and enhanced phase mixing of torsional Alfvén waves in open structures in the solar corona with exponentially diverging magnetic field lines and a gravitationally stratified atmosphere. Wave based heating mechanisms have always been part of the discussion surrounding the coronal heating problem (Arregui 2015) and since the advent of space-based EUV imagers a large variety of waves and oscillations have been detected in the solar atmosphere (Parnell \& De Moortel 2012). In particular, torsional Alfvén waves have recently been directly detected in a magnetic pore at the photosphere (Stangalini et al. 2021). It was shown in (Soler et al. 2017) that torsional Alfvén waves of intermediate frequencies are able to penetrate to the corona whilst low frequency waves are reflected and high frequency waves are absorbed in the chromosphere due to ion-neutral damping. Furthermore it was shown that the transmission of torsional Alfvén waves to the corona is improved in a magnetic field with exponentially diverging field lines.

Alfvén waves are believed to be viable transporters of the nonthermal energy required to heat the solar corona (Mathioudakis et al. 2013) but they are notoriously difficult to dissipate due to the high conductivity of the corona, (Tsiklauri 2009). For this reason dissipation must occur over scales smaller than typical MHD scales. Mechanisms for generating these small length scales typically rely

^ E-mail: c.boocock@qmul.ac.uk on inhomogeneities in the corona, (De Moortel et al. 2008). Phase mixing was first proposed by (Heyvaerts \& Priest 1983) as a mechanism for enhancing the viscous and ohmic dissipation of Alfvén waves in loops and open regions of the solar corona. When shear Alfvén waves on neighboring magnetic surfaces propagate at different speeds they move out of phase with each other. This leads to the build up of strong gradients perpendicular to the direction of propagation that allows dissipation of the wave through shear viscosity or resistivity heating the plasma.

The efficiency of phase mixing is strongly affected by both the magnetic field configuration and the density stratification of the plasma, (De Moortel et al. 2000). Enhanced phase mixing occurs in magnetic field structures with exponentially diverging field lines and significantly increases the dissipation rate of Alfvén waves. In this case the magnetic field strength decreases exponentially with height and therefore, so does the Alfvén speed. The effect is that as Alfvén waves propagate upwards, their wavelengths are decreased and this leads to stronger transverse gradients, which enhances the effect of phase mixing. Conversely density stratification causes wavelengths to increase as the waves propagate upwards and this reduces the effects of phase mixing (Smith et al. 2007).

In this paper we consider the effect of enhanced phase mixing on torsional Alfvén waves propagating upwards in an exponentially diverging field line structure with a transverse density gradient and stratified atmosphere. An analytic formulation for the evolution of shear Alfvén waves in a two-dimensional open magnetic field configuration is given by (Ruderman et al. 1998) using the WKB approx- 
imation. In (Smith et al. 2007) a corrected form of this analytical formulation was validated using a 2.5D visco-resistive linear MHD code and the dependency of the heating length scale on the magnetic scale heights was investigated. The conclusion was that the dissipation of Alfvén waves is strongly dependent on the field line divergence.

In both (Ruderman et al. 1998) and (Smith et al. 2007) it is assumed that the wavelength of Alfvén waves is much smaller than the characteristic scale of variation of the equilibrium parameters. This allows the WKB approximation to be used and the effect of wave reflection to be neglected. Wave reflection means that part of the Alfvén wave is reflected back along the same field line due to the longitudinal inhomogeneity in the Alfvén speed, as described, for example, in (Morton et al. 2015).

In (Ruderman \& Petrukhin 2017) and (Petrukhin et al. 2018) nonreflective magnetic plasma configurations are considered; these are a planar and cylindrical configurations respectively. Exponentially divergent magnetic field lines are still used but the density profiles are altered to keep the configuration non-reflective. Phase mixed solutions for Alfvén waves propagating in these configurations are derived without using the WKB approximation. For these formulations the wavelength need not be much smaller than the characteristic scale of variation, it can be of the same order. The density profiles used however are only possible within a very restrictive range of parameters. To consider a more general configuration the effects of wave reflection must be included in the solutions.

In (Ruderman \& Petrukhin 2018) the WKB approximation is once again used to find the phase mixing solution of torsional Alfvén waves propagating in a cylindrically symmetric, potential magnetic field with exponentially diverging field lines, a radial density gradient and a stratified atmosphere. In this formulation the wavelength is not assumed to be much smaller than the characteristic scale of variation. Instead conditions are found in which the effect of wave reflection can be considered weak and neglected.

In this paper we begin by considering the analytic formulation for the propagation and phase mixing of torsional Alfvén waves. The next section section 2 is split into three parts. In section 2.1 we go through the derivation of the analytic solution presented in (Ruderman \& Petrukhin 2018) making a simple correction towards the end. In section 2.2 we describe how the wave energy flux of the Alfvén wave, $\Pi$, can be calculated over successive magnetic surfaces. Then in section 2.3 we describe the numerical setup for an equilibrium with divergent magnetic field lines and an exponentially stratified density profile which we use in our numerical calculations.

In section 3 we present a numerical code, named TAWAS and written in IDL, that calculates the analytical solution over a coordinate grid in radius, $r$ and height, $z$. We use TAWAS firstly to demonstrate the effects of both wave reflection, and of our correction to the solution in section 4 . Then in section 5 we use TAWAS to perform parameter studies to determine the conditions under which the damping of torsional Alfvén waves is strongest.

In section 6 we introduce a second code Wigglewave, that directly solves the linearised governing equations for torsional Alfén wave propagation and is fourth order accurate in space and time. We compare the results from TAWAS and Wigglewave to test the validity of the analytical solution both within and beyond the limits of the WKB approximation. Our aim is to determine whether the wave damping caused by enhanced phase mixing is stronger or weaker than the analytic solution suggests. Finally in section 7 we discuss our results and summarise our findings.

The overall aim of this work is to correct and validate the analytic solution presented in (Ruderman \& Petrukhin 2018) and to identify the conditions under which the WKB approximation, and by extension the analytic formula, is valid. In particular we will see whether Alfvén wave damping is stronger or weaker than predicted by this formula beyond the limits of the WKB approximation.

\section{ANALYTIC SOLUTIONS}

\subsection{Solution Formulation}

We begin by considering the analytic solution for the propagation and phase-mixing of torsional Alfvén waves through a viscous plasma that is in axisymmetric equilibria. Such a solution is presented in $(\mathrm{Ru}-$ derman \& Petrukhin 2018) and we will follow this solution closely, correcting a mistake in the solution towards the end of the derivation, as confirmed by (Ruderman 2021).

The only dissipative process considered here is viscosity whilst resistivity is neglected. In (Heyvaerts \& Priest 1983) it is shown that the kinematic viscosity and magnetic diffusivity are additive in the dissipative term of the wave equation. As kinematic viscosity and magnetic diffusivity are of the same order in the corona neglecting resistivity can only reduce the efficiency of wave damping by a factor of unity; on the other hand this allows us to simplify the analysis by eliminating the magnetic perturbation from the governing equations.

As our plasma is in axisymmetric equilibria all of the equilibrium quantities are dependent on only $r$ and $z$ in cylindrical coordinates. We consider an equilibrium magnetic field without azimuthal component $\mathbf{B}_{\mathbf{0}}=\left(B_{r}, 0, B_{z}\right)$. As the plasma beta in the corona is very low, we assume a negligible pressure. Our magnetic field must therefore be a force-free, axisymmetric magnetic field without azimuthal component. This type of field is always potential and therefore we can express the radial and vertical components $B_{r}$ and $B_{z}$ in terms of the magnetic potential, $\phi$, and magnetic flux, $\psi$,

$$
\frac{B_{r}}{B_{0}}=\frac{\partial \phi}{\partial r}=-\frac{H}{r} \frac{\partial \psi}{\partial z}, \quad \frac{B_{z}}{B_{0}}=\frac{\partial \phi}{\partial z}=\frac{H}{r} \frac{\partial \psi}{\partial r},
$$

where $B_{0}$ is a constant characterizing the magnetic field strength and $H$ is the magnetic scale height which characterizes the divergence of the magnetic field lines with height. Below we use the curvilinear coordinate system $(\phi, \psi)$ in the plane $\theta=$ constant. By definition $\phi$ and $\psi$ are orthogonal with $\phi$ coordinate lines coinciding with the magnetic field lines and $\psi$ coordinate lines being orthogonal to the magnetic field lines. This coordinate system is sketched in fig. 1.

We begin our analysis with the MHD equations for a zero beta plasma,

$$
\begin{aligned}
\frac{\partial \rho}{\partial t}+(\mathbf{v} \cdot \nabla) \rho & =-\rho(\nabla \cdot \mathbf{v}) \\
\left.\rho\left(\frac{\partial \mathbf{v}}{\partial t}+(\mathbf{v} \cdot \nabla) \mathbf{v}\right)\right) & =-\frac{1}{\mu_{0}} \mathbf{B} \times(\nabla \times \mathbf{B})+\nabla \cdot(\rho v \nabla \mathbf{v}), \\
\left.\frac{\partial \mathbf{B}}{\partial t}+(\mathbf{v} \cdot \nabla) \mathbf{B}\right) & =(\mathbf{B} \cdot \nabla) \mathbf{v}-\mathbf{B}(\nabla \cdot \mathbf{v}),
\end{aligned}
$$

We linearise these equations and consider purely azimuthal perturbations to the velocity and magnetic field of the plasma, so that only the $\theta$-components of the velocity and magnetic field perturbation, $v=v_{\theta}$ and $b=b_{\theta}$, are non-zero. This results in torsional Alfvén waves that are incompressible and are described by the $\theta$-components of the momentum and induction equation, 


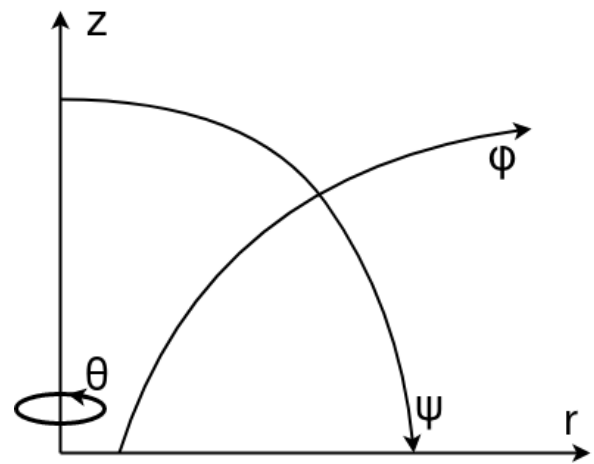

Figure 1. A sketch of the cylindrical and curvilinear coordinate systems. In the cylindrical system we have the height, $z$ and the radius, $r$ whereas in the curvilinear field aligned coordinate system we have $\phi$, which varies along field lines and $\psi$, which varies across field lines. Both coordinate systems include the azimuthal coordinate $\theta$.

$$
\begin{aligned}
\rho \frac{\partial v}{\partial t} & =\frac{1}{r \mu_{0}}\left(\mathbf{B}_{\mathbf{0}} \cdot \nabla(r b)\right)+\frac{1}{r} \frac{\partial}{\partial r}\left(\rho v r \frac{\partial v}{\partial r}\right)+\frac{\partial}{\partial z}\left(\rho v \frac{\partial v}{\partial z}\right), \\
\frac{\partial b}{\partial t} & =r \mathbf{B}_{\mathbf{0}} \cdot \nabla\left(\frac{v}{r}\right),
\end{aligned}
$$

where $\rho$ is the density, $\mu_{0}$ is the vacuum permeability, $v$ is the kinematic viscosity, which is taken to be constant, and $B_{0} \cdot \nabla=$ $B_{r} \partial_{r}+B_{z} \partial_{z}$. We now make the substitution $v=r u$ where $u$ is the angular velocity. It is shown in (Petrukhin et al. 2018) that we can simplify the viscous terms in eq. (5) by making the approximation,

$\frac{1}{r} \frac{\partial}{\partial r}\left(\rho v r \frac{\partial v}{\partial r}\right)+\frac{\partial}{\partial z}\left(\rho v \frac{\partial v}{\partial z}\right) \approx \rho v r\left(\frac{\partial^{2} u}{\partial r^{2}}+\frac{\partial^{2} u}{\partial z^{2}}\right)$,

we can then take the time derivative of eq. (5) and then eliminate $b$ using eq. (6) to obtain the wave equation,

$\frac{\partial^{2} u}{\partial t^{2}}=\frac{1}{\mu_{0} \rho r^{2}} \mathbf{B}_{\mathbf{0}} \cdot \nabla\left(r^{2} \mathbf{B}_{\mathbf{0}} \cdot \nabla u\right)+v\left(\frac{\partial}{\partial t} \frac{\partial^{2} u}{\partial r^{2}}+\frac{\partial}{\partial t} \frac{\partial^{2} u}{\partial z^{2}}\right)$.

We continue to follow (Petrukhin et al. 2018) and now switch to using the curvilinear magnetic field coordinates $\phi$ and $\psi$ as independent variables, our partial derivatives become,

$$
\begin{aligned}
\frac{\partial}{\partial r} & =\frac{B_{r}}{B_{0}} \frac{\partial}{\partial \phi}+\frac{r}{H} \frac{B_{z}}{B_{0}} \frac{\partial}{\partial \psi}, \\
\frac{\partial}{\partial z} & =\frac{B_{z}}{B_{0}} \frac{\partial}{\partial \phi}-\frac{r}{H} \frac{B_{r}}{B_{0}} \frac{\partial}{\partial \psi}, \\
\mathbf{B}_{0} \cdot \nabla & =\frac{B^{2}}{B_{0}} \frac{\partial}{\partial \phi},
\end{aligned}
$$

where $B$ is the field strength of our equilibrium magnetic field $\mathbf{B}_{\mathbf{0}}$. By assuming that derivatives in the $\psi$ direction, perpendicular to field lines, dominate over derivatives in the $\phi$ direction, along field lines, we can make the approximation,

$\frac{\partial^{2} u}{\partial r^{2}}+\frac{\partial^{2} u}{\partial z^{2}}=\frac{r^{2} B^{2}}{H^{2} B_{0}^{2}} \frac{\partial^{2} u}{\partial \psi^{2}}$,

which transforms our wave equation into:

$$
\frac{\partial^{2} u}{\partial t^{2}}=\frac{V_{A}^{2}}{r^{2}} \frac{\partial}{\partial \phi}\left(\frac{r^{2} B^{2}}{B_{0}^{2}} \frac{\partial u}{\partial \phi}\right)+\frac{v r^{2} B^{2}}{H^{2} B_{0}^{2}} \frac{\partial^{3} u}{\partial t \partial \psi^{2}},
$$

where

$V_{A}=\frac{B}{\sqrt{\mu_{0} \rho}}$

is the Alfvén velocity. We now look for a solution to eq. (13) of the form,

$u(t, \phi, \psi)=\frac{v_{0}}{H} A(\phi, \psi) \Phi(t, h(\phi, \psi), \psi)$,

where $v_{0}$ is a constant of velocity. The functions $A(\phi, \psi)$ and $h(\phi, \psi)$ can be chosen arbitrary, we define them as:

$h(\phi, \psi)=B_{0} V_{0} \int_{\phi_{1}(\psi)}^{\phi} \frac{d \phi^{\prime}}{B V_{A}}, \quad A(\phi, \psi)=A_{0}(\psi) \frac{H}{r}\left(\frac{\rho_{0}}{\rho}\right)^{1 / 4}$,

where $V_{0}=B_{0} / \sqrt{\mu_{0} \rho_{0}}, \rho_{0}$ is the density at the origin and $\phi_{1}(\psi)$ $A_{0}(\psi)$ are arbitrary functions with the condition that $A_{0}(\psi) / r$ is nonzero in the limit $\psi \rightarrow 0$. With these functions defined, substituting eq. (15) into eq. (13) gives the following equation for $\Phi$ :

$\frac{\partial^{2} \Phi}{\partial t^{2}}-V_{0}^{2} \frac{\partial^{2} \Phi}{\partial h^{2}}=\frac{v r^{2} B^{2}}{H^{2} B_{0}^{2}} \frac{\partial \Xi}{\partial t}+\frac{V_{A}^{2} \Phi}{A r^{2}} \frac{\partial}{\partial \phi}\left(\frac{r^{2} B^{2}}{B_{0}^{2}} \frac{\partial A}{\partial \phi}\right)$,

where

$$
\begin{aligned}
\Xi= & \frac{1}{A} \frac{\partial^{2}(A \Phi)}{\partial \psi^{2}}+\frac{2}{A} \frac{\partial h}{\partial \psi} \frac{\partial}{\partial \psi}\left(A \frac{\partial \Phi}{\partial h}\right) \\
& +\frac{\partial^{2} h}{\partial \psi^{2}} \frac{\partial \Phi}{\partial \psi}+\left(\frac{\partial h}{\partial \psi}\right)^{2} \frac{\partial^{2} \Phi}{\partial h^{2}} .
\end{aligned}
$$

The terms on the RHS of eq. (17) prescribe the wave damping from viscous dissipation and wave reflection respectively. In order to apply the WKB approximation it is assumed that both the terms on the RHS of eq. (17) are much smaller than the terms on the LHS. We now assume Alfvén waves are driven at the lower boundary, representing the base of the corona, and then propagate along magnetic field lines. Considering harmonic waves and taking all time-dependant quantities as proportional to $e^{i \omega t}$ reduces eq. (17) to,

$V_{0}^{2} \frac{\partial^{2} \Phi}{\partial h^{2}}+\omega^{2} \Phi=\frac{i \omega v r^{2} B^{2}}{H^{2} B_{0}^{2}} \Xi-\frac{V_{A}^{2} \Phi}{A r^{2}} \frac{\partial}{\partial \phi}\left(\frac{r^{2} B^{2}}{B_{0}^{2}} \frac{\partial A}{\partial \phi}\right)$.

Our assumption that wave damping is weak allows the use of the following scaled quantities in our wave equation,

$\tilde{h}=\epsilon h, \quad \tilde{v}=\operatorname{Re} v, \quad \tilde{\phi}=\epsilon \phi$,

where the scaling parameter $\epsilon \ll 1$ and $\operatorname{Re}=H V_{0} / v$ is the Reynolds number, see (Ruderman \& Petrukhin 2018) for details. Substituting these scaled quantities transforms eq. (19) once again,

$\epsilon^{2} V_{0}^{2} \frac{\partial^{2} \Phi}{\partial \tilde{h}^{2}}+\omega^{2} \Phi=\frac{i \omega \tilde{v} r^{2} B^{2}}{H^{2} B_{0}^{2} R e} \tilde{\Xi}-\frac{\epsilon V_{A}^{2} \Phi}{A r^{2}} \frac{\partial}{\partial \tilde{\phi}}\left(\frac{r^{2} B^{2}}{B_{0}^{2}} \frac{\partial A}{\partial \phi}\right)$. 
At this stage the standard WKB method is used to look for a solution in the form,

$\Phi=Q(\tilde{h}, \psi) \exp i \epsilon^{-1} \Theta(\tilde{h}, \psi)$,

where $\tilde{\Xi}$ is simply $\Xi$ but with $\tilde{h}$ substituted for $h$. Substituting this expression into eq. (21) and using the expression for $\tilde{\Xi}$ gives:

$$
\begin{aligned}
\epsilon^{2} \frac{\partial^{2} Q}{\partial \tilde{h}^{2}}+ & 2 i \epsilon \frac{\partial Q}{\partial \tilde{h}} \frac{\partial \Theta}{\partial \tilde{h}}+i \epsilon Q \frac{\partial^{2} \Theta}{\partial \tilde{h}^{2}}-Q\left(\frac{\partial \Theta}{\partial \tilde{h}}\right)^{2}+\frac{\omega^{2}}{V_{0}^{2}} Q \\
= & -\frac{i \epsilon^{-2} \omega \tilde{v} r^{2} B^{2}}{H^{2} B_{0}^{2} V_{0}^{2} R e} Q\left[\left(\frac{\partial \tilde{h}}{\partial \psi}\right)^{2}\left(\frac{\partial \Theta}{\partial \tilde{h}}\right)^{2}+O(\epsilon)\right] \\
& -\frac{\epsilon V_{A}^{2}}{A r^{2} V_{0}^{2}} Q \frac{\partial}{\partial \tilde{\phi}}\left(\frac{r^{2} B^{2}}{B_{0}^{2}} \frac{\partial A}{\partial \phi}\right)
\end{aligned}
$$

Now terms of the same order are collected. Assuming that the first term on the RHS is smaller than the two largest terms on the LHS, terms of the order unity are collected. This is called the approximation of geometrical optics and determines the shape of the rays along which waves propagate. This gives us an equation for $\Theta$ :

$$
\left(\frac{\partial \Theta}{\partial \tilde{h}}\right)^{2}=\frac{\omega^{2}}{V_{0}^{2}},
$$

which when solved for waves propagating in the positive $\phi$ direction gives:

$\Theta=\frac{\tilde{h} \omega}{V_{0}}$.

Now the terms of order $\epsilon$ are collected. This is called the approximation of physical optics and determines the spatial evolution of the wave amplitude. The first term on the RHS which describes resistive damping due to phase mixing should be included in this contribution hence we set $R e=\epsilon^{-3}$.

This is where our correction is made and our solution diverges from that given in (Ruderman \& Petrukhin 2018). In eq.(18) of (Ruderman $\&$ Petrukhin 2018) when the terms of order $\epsilon$ are collected all the terms are divide through $i$ except for the second term on the RHS. Here we correct this by dividing all terms by $i$. This gives us,

$$
\begin{aligned}
2 \frac{\partial Q}{\partial \tilde{h}} \frac{\partial \Theta}{\partial \tilde{h}}+Q \frac{\partial^{2} \Theta}{\partial \tilde{h}^{2}}= & -\frac{\omega \tilde{v} r^{2} B^{2}}{H^{2} B_{0}^{2} V_{0}^{2}} Q\left(\frac{\partial \tilde{h}}{\partial \psi}\right)^{2}\left(\frac{\partial \Theta}{\partial \tilde{h}}\right)^{2} \\
& +\frac{i V_{A}^{2}}{A r^{2} V_{0}^{2}} Q \frac{\partial}{\partial \tilde{\phi}}\left(\frac{r^{2} B^{2}}{B_{0}^{2}} \frac{\partial A}{\partial \phi}\right) .
\end{aligned}
$$

Returning to non-scaled variables now gives the following ordinary differential equation for $\mathrm{Q}$ :

$$
\frac{\partial Q}{\partial \phi}=(i R-\Upsilon) Q
$$

where,

$$
\begin{aligned}
\Upsilon & =\frac{\omega^{2} v}{2 V_{0}^{3} G^{2}}\left(\frac{\partial h}{\partial \psi}\right)^{2}, \\
R & =\frac{G V_{0}}{2 \omega H^{2}} \frac{\partial}{\partial \phi}\left(\frac{r^{2} B^{2}}{B_{0}^{2}} \frac{\partial}{\partial \phi} G\right),
\end{aligned}
$$

and $G=A / A_{0}$. We also impose the condition:

$Q=1$ at $\phi=\phi_{1}(\psi)$.

As with (Ruderman \& Petrukhin 2018) we can now use eqs. (15), (16), (22) and (25) and recall that the perturbations are proportional to $e^{-i \omega t}$ to obtain:

$v=v_{0} W \exp \left[i \omega\left(h / V_{0}-t\right)\right], \quad W=Q A_{0}(\psi)\left(\frac{\rho_{0}}{\rho}\right)^{1 / 4}$.

For the magnetic field perturbation we can use eqs. (6), (16) and (31) and the relation eq. (11) to yield,

$b=v_{0} B\left[\frac{i r B}{\omega B_{0}} \frac{\partial(W / r)}{\partial \phi}-\frac{W}{V_{A}}\right] \exp \left[i \omega\left(h / V_{0}-t\right)\right]$.

These solutions differ from those given in (Ruderman \& Petrukhin 2018) as $Q$ and consequently $W$ now have different values, furthermore they are now complex valued (Ruderman 2021).

\subsection{Wave Energy Flux Calculation}

In order to characterise the efficiency of wave damping we follow the example in (Ruderman \& Petrukhin 2018) being careful to account for the fact that $Q$ and $W$ are now complex valued. We calculate the wave energy flux across magnetic surfaces defined by $\phi=$ constant. Each surface is uniquely identified by its height of intersection with the $z$-axis, hence we can define the energy flux $\Pi$ as a function of height $z$. Multiplying eq. (5) by $v$, eq. (6) by $b / \mu_{0}$ and adding the results we obtain,

$$
\begin{aligned}
\frac{\partial}{\partial t}\left(\frac{\rho v^{2}}{2}+\frac{b^{2}}{2 \mu_{0}}\right)= & \frac{1}{\mu_{0}} \nabla \cdot\left(\mathbf{B}_{\mathbf{0}} v b\right) \\
& +\frac{v}{r} \frac{\partial}{\partial r}\left(\rho v r \frac{\partial v}{\partial r}\right)+v \frac{\partial}{\partial z}\left(\rho v \frac{\partial v}{\partial z}\right) .
\end{aligned}
$$

The expression in the parenthesis on the LHS is the wave energy density, whilst $\mathbf{B}_{\mathbf{0}} v b / \mu_{0}$ is the density of wave energy flux. When $v=0$ this is the equation for wave energy conservation. The density of wave energy flux is directed along the field lines. We now want to obtain an expression for the density of wave energy flux averaged over a wave period.

To obtain physical quantities we need to take the real parts of $v$ and $b$ as given in eq. (31) and eq. (32). It is however no longer possible to reduce this quantity to a simple expression in terms of $W$, for example by evaluating $\partial(W / r) / \partial \phi$ we see that the expansion of $b$ is,

$$
\begin{gathered}
b=-v_{0} W\left[\frac{i B^{2}}{\omega B_{0}}\left[\frac{1}{Q} \frac{\partial Q}{\partial \phi}+\frac{1}{4 \rho} \frac{\partial \rho}{\partial \phi}+\frac{1}{r} \frac{\partial r}{\partial \phi}\right]+\sqrt{\mu_{0} \rho}\right] \\
\exp \left[i \omega\left(h / V_{0}-t\right)\right] .
\end{gathered}
$$

Simultaneously we are faced with the issue that in order to evaluate the density of wave energy flux for our simulation results we will have to use the values of $v$ and $b$ directly. For both of these reasons we decide to calculate wave energy flux through each surface using the wave envelopes for $v$ and $b$. By using the assumption that $v$ and $b$ are in anti-phase we can conclude that the density of wave energy flux averaged over a wave period is,

$-\frac{B}{\mu_{0}}\langle v b\rangle=\frac{B}{2 \mu_{0}} v_{p} b_{p}$, 
where $v_{p}$ and $b_{p}$ are the maximum values of the wave envelopes for $v$ and $b$ respectively. We now define $\Sigma$ as the magnetic surface defined by $\phi_{*}=$ constant, that intersect the $z$-axis at $z_{*}$. We assume that the waves propagate in the magnetic tube bounded by the surface $\psi=\psi_{b}$. The wave energy flux through $\Sigma$ is then the density of wave energy flux integrated over the part of $\Sigma$ in the magnetic tube, we denote this part as $\Sigma_{b}$. It is shown in (Ruderman \& Petrukhin 2018) that,

$d \Sigma=\frac{H B_{0}}{B} d \psi d \theta$.

Now using eq. (35) and eq. (36) we obtain that the average energy flux through the surface $\Sigma_{b}$ is,

$\Pi(z)=-\frac{1}{\mu_{0}} \int_{\Sigma_{b}} B\langle v b\rangle d \Sigma=\pi \frac{H B_{0}}{\mu_{0}} \int_{0}^{\psi_{b}} v_{p} b_{p} d \psi$.

\subsection{Numerical Setup}

We can now use our analytic solution to solve for the propagation of torsional Alfvén waves in a potential magnetic field with exponentially diverging field lines and a stratified density profile. First we must define the magnetic structure and density profile, our setup is identical to that given in (Ruderman \& Petrukhin 2018).

We consider a magnetic field that is potential, axisymmetric and has no azimuthal component. The field must therefore satisfy the vector Laplace equation $\nabla^{2} \mathbf{B}_{\mathbf{0}}=0$ in cylindrical coordinates, the solution to this equation is outlined in appendix A. Solving this equation with the criteria that the field strength decreases with height exponentially according to our magnetic scale height $H$, defines our magnetic field as,

$B_{r}=B_{0} e^{-z / H} J_{1}(r / H), \quad B_{z}=B_{0} e^{-z / H} J_{0}(r / H)$,

where $J_{0}$ and $J_{1}$ are Bessel functions of the first kind and of zero and first order respectively. The functions $\phi$ and $\psi$ are then given by,

$\phi=-H e^{-z / H} J_{0}(r / H), \quad \psi=r e^{-z / H} J_{1}(r / H)$.

We define our gravitationally stratified atmosphere as follows,

$\rho=\hat{\rho}(\psi) e^{-z / H_{\rho}}=\hat{\rho}(\psi) e^{-\alpha z / H}$,

where the density scale height $H_{\rho}=k_{B} T_{0} / m g, k_{B}$ is Boltzmann's constant, $T_{0}$ is the temperature in the corona and is constant, $m \approx$ $0.6 m_{p}$ in the corona, $m_{p}$ is the proton mass, $g$ is the solar surface gravity, $\alpha=H / H_{\rho}$ and $\hat{\rho}(\psi)$ is an arbitrary function that defines the density variation across magnetic field lines.

We use $\hat{\rho}(\psi)$ to define a central higher density tube enclosed within the field line defined by $\psi=\psi_{b}$, we will study the propagation of torsional Alfvén waves within this tube. We want this tube to have a density gradient transverse to the propagation of Alfvén waves along magnetic field lines so that we can study the effect of phase mixing. We do this by defining $\hat{\rho}(\psi)$ to be,

$\hat{\rho}(\psi)=\frac{\rho_{0}}{\zeta} \begin{cases}1+(\zeta-1)\left(1-\psi / \psi_{b}\right)^{2}, & \psi \leq \psi_{b} \\ 1, & \psi \geq \psi_{b}\end{cases}$

where $\zeta$ is the density contrast between the centre and exterior of the magnetic tube $\hat{\rho}(0) / \hat{\rho}\left(\psi_{b}\right)$. An example of what the density

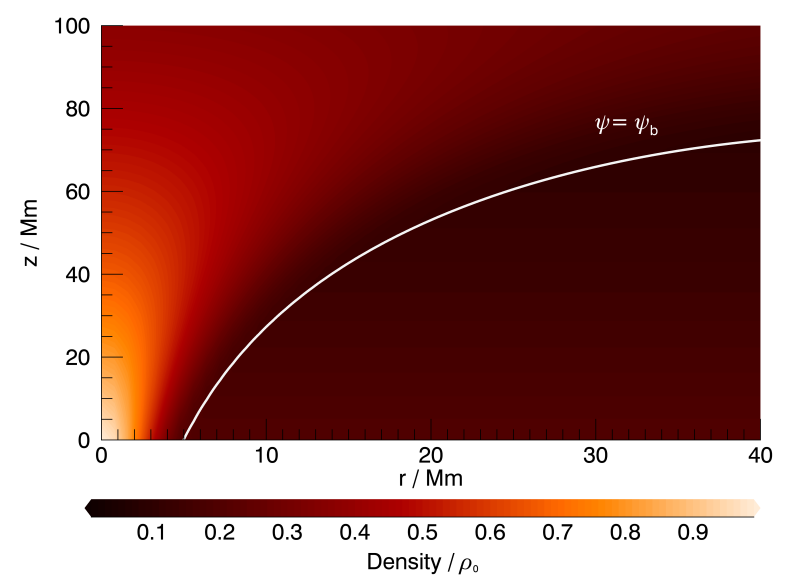

Figure 2. An example of the density contrast for this setup, brighter areas represent a higher density, the white contour shows the boundary of the central high density tube. For this particular example the scale height used are $H=20 \mathrm{Mm}$ and $H_{\rho}=100 \mathrm{Mm}$.

structuring looks like is given in fig. 2 the tube boundary at $\psi=\psi_{b}$ is highlighted for clarity.

Finally we define our wave driving, we would like to drive our waves from the lower boundary of the domain however strictly speaking we should be driving waves from a magnetic surface defined by $\phi=$ const, let us consider the magnetic surface through the coordinate origin $r=0, z=0$ which has a value $\phi=-H$. Let this surface intersect $\psi=\psi_{b}$ at $r=r_{0}$ and $z=z_{0}$, from eq. (39) we then have,

$\psi_{b}=r_{0} \frac{J_{1}\left(r_{0} / H\right)}{J_{0}\left(r_{0} / H\right)}, \quad z_{0}=-H \ln \left(J_{0}\left(r_{0} / H\right)\right)$.

By taking $r_{0} \ll H$ we can approximate the Bessel functions by the leading terms in their Taylor series, $J_{0}\left(r_{0} / H\right) \approx 1, J_{1}\left(r_{0} / H\right) \approx$ $r_{0} / 2 H$. This leads us to,

$\psi_{b} \approx r_{0}^{2} / 2 H, \quad z_{0} \approx 0$.

This implies that the magnetic surface $\phi=-H$ roughly coincides with the lower boundary $z=0$ within the confines of the tube meaning we can treat the lower boundary as a magnetic surface. The radius $r_{0}$ is where the tube boundary $\psi=\psi_{b}$ intersects the lower boundary and defines the width of the tube. We can therefore drive our Alfven waves from the lower boundary $z=0$ and within the tube structure $r \leq r_{0}$, we define our wave driving by,

$u=\left\{\begin{array}{ll}u_{0}\left(1-\frac{r^{2}}{r_{0}^{2}}\right) e^{-i \omega t}, & r \leq r_{0} \\ 0, & r \geq r_{0}\end{array} \quad\right.$ at $z=0$

From this expression for the angular velocity $u$ we can find the linear velocity $v=r u$ which we will use when driving waves in our simulations. We can calculate $A_{0}(\psi)$ which is required for the analytic solution. By comparing this expression for $u$ with eq. (31), noticing that $h=0$ at $z=0$ and using the relation $r^{2} / r_{0}^{2}=\psi / \psi_{b}$, we have,

$A_{0}(\psi)= \begin{cases}\left(\frac{\hat{\rho}}{\rho}\right)^{1 / 4}\left(\frac{\psi}{\psi_{b}}\right)^{1 / 2}\left(1-\frac{\psi}{\psi_{b}}\right), & \psi \leq \psi_{b} \\ 0, & \psi \geq \psi_{b}\end{cases}$ 
In what follows we consider the propagation of Alfvén waves in a typical coronal plume or divergent coronal loop structures. We set our characteristic values for field strength and density as $B_{0}=0.001$ $\mathrm{T}$, and $\rho_{0}=1.66 \times 10^{-12} \mathrm{~kg} \mathrm{~m}^{-3}$ (which corresponds to a number density of approximately $n_{0}=1 \times 10^{15} \mathrm{~m}^{-3}$ ). This gives us an Alfvén speed of $V_{0} \approx 700 \mathrm{~m} \mathrm{~s}^{-1}$. We fix the initial tube radius to be $r_{0}=5 \mathrm{Mm}$ and the density contrast to be $\zeta=5$ for all cases. We set the amplitude of our Alfvén wave driving at the lower boundary, representing the base of the corona, to have a maximum value of about $40 \mathrm{~km} \mathrm{~s}^{-1}$, based on (Banerjee et al. 1998) and (Doyle et al. 1999), this is equivalent to setting $u_{0}=100 \mathrm{~km} \mathrm{~s}^{-1}$.

The parameters that we vary across different cases are the magnetic and density scale heights, $H$ and $H_{\rho}$, the wave period $T$ and the kinematic viscosity $v$. The magnetic and density scale heights used are between 20 and $100 \mathrm{Mm}$. The magnetic scale height depends very much on the structure being considered, however, considering the temperature of the corona is 1-2 MK, see (Aschwanden 2004), we would expect the density scale height be somewhere between 50-100 Mm. The wave periods considered are between 10 and 120 seconds as we expect shorter period waves to be absorbed in the chromosphere and longer period waves to be reflected as discussed in (Soler et al. 2017). The values we use for kinematic viscosity range between $v=1 \times 10^{4}$ and $5 \times 10^{7} \mathrm{~m}^{2} \mathrm{~s}^{-1}$, these values are several orders of magnitude greater than would be expected from classical plasma theory (Braginskii 1965). This anomalous viscosity could be caused by turbulence as described in (Liu et al. 2014), possibly triggered by the Kelvin-Helmholtz instability as discussed in (Soler et al. 2021), indeed numerical evidence for such values is presented in (Tsiklauri 2006).

\section{ANALYTIC SOLVER: TAWAS}

Using this numerical setup we proceed to calculate solutions for torsional Alfvén wave propagation for different cases, with each case using different values for the magnetic and density scale heights, $H$ and $H_{\rho}$, the wave period $T$ and the kinematic viscosity $v$. We calculate solutions using our analytical formula and an IDL script written by the authors called TAWAS (Torsional Alfvén wave analytic solution).

TAWAS calculates the analytic solutions for $v$ and $b$ over a uniform numerical grid using the analytic formula described in section 2.1 and then calculates the wave energy flux $\Pi(z)$ as a function of the height using eq. (37) in section 2.2. The script for this code can be found at https://github.com/calboo/TAWAS under the filename tawas.pro, along with a description of how the calculations are performed.

Every time TAWAS was used in this study the resolution was set to $500 \times 2000$ in the $r$ and $z$ directions respectively. The domain height was fixed at $z_{\max }=100 \mathrm{Mm}$ but the domain radius $r_{\max }$ was varied depending on $H$ to allow for maximal resolution in each case.

Examples of the output from TAWAS are shown in figs. 3 to 5, these were produced from a case with the parameters $H=20 \mathrm{Mm}$, $H_{\rho}=100 \mathrm{Mm}, T=60 \mathrm{~s}$ and $v=5 \times 10^{7} \mathrm{~m}^{2} \mathrm{~s}^{-1}$. fig. 3 and fig. 4 show the velocity and magnetic field evolution of the wave over the domain and fig. 5 shows the normalised wave energy flux, $\Pi(z)$, plotted against height.

We can see the damping effects of both reflection and phase mixing in fig. 5. Reflective damping occurs lower in the domain and its effect decreases with height whereas the damping caused by phase mixing is initially small and its effect increases with height. This is because the reflection and phase mixing of Alfvén waves depends

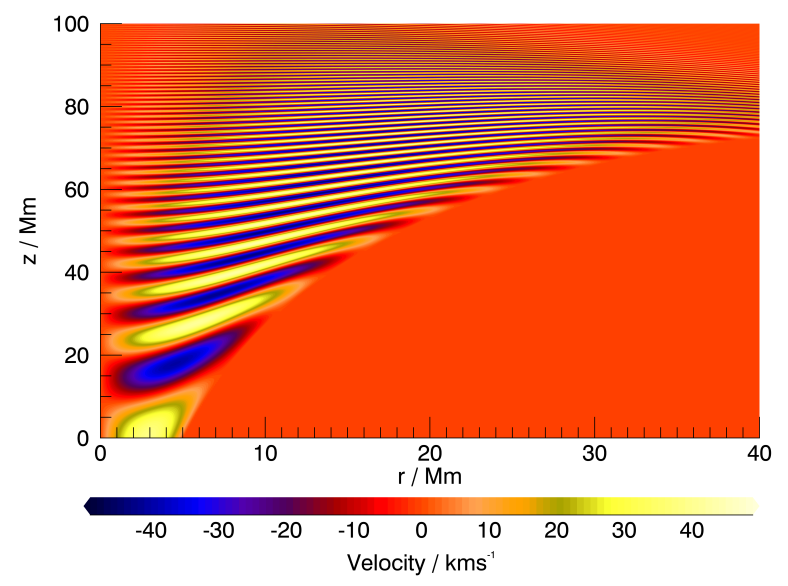

Figure 3. An example of the velocity output from TAWAS. This contour plot shows the azimuthal velocity $v$ of the plasma as the torsional Alfvén wave propagates through the central tube. Note that at higher heights the wavelength become very small so the wave fronts can only be resolved fully when zoomed in.

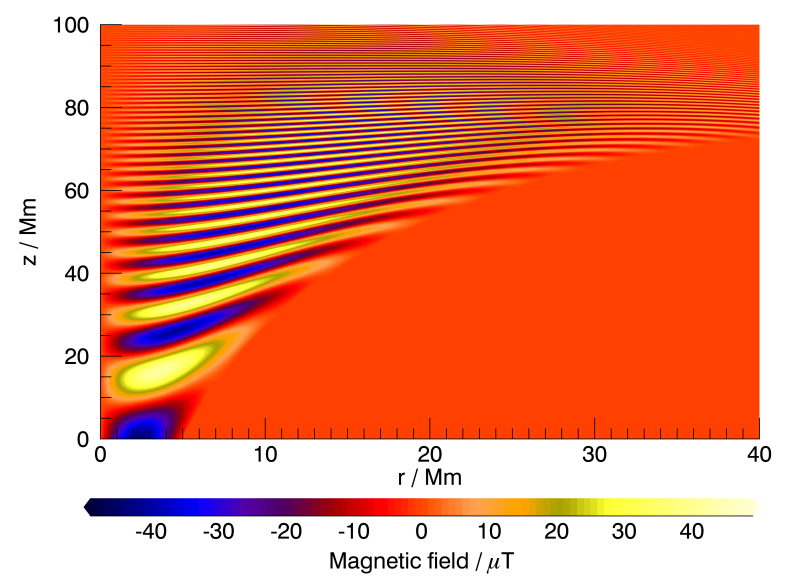

Figure 4. An example of the magnetic field output from TAWAS. This contour plot shows the azimuthal magnetic field $b$ of the plasma as the torsional Alfvén wave propagates through the central tube. Note that at higher heights the wavelength become very small so the wave fronts can only be resolved fully when zoomed in.

on their wavelength. Longer wavelengths are reflected more strongly and shorter wavelengths undergo more phase mixing due to stronger transverse gradients that lead to greater viscous dissipation (Smith et al. 2007). In this case the divergence of the magnetic field lines causes the Alfvén velocity and therefore the wavelength to decrease with height thus reducing the effect of reflection and enhancing the effect of phase mixing as height increases.

\section{EFFECT OF CORRECTION TO FORMULA}

Now that we have a tool for numerically solving the analytic formula, we want to show the effect of our correction to the formula. Our correction only affects damping through wave reflection. In (Ruderman $\&$ Petrukhin 2018) the effect of reflection was neglected entirely in 


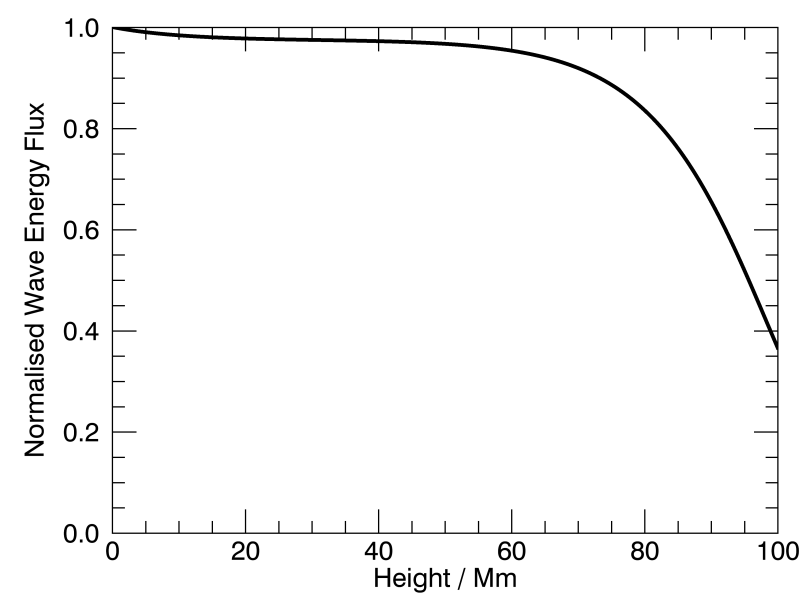

Figure 5. An example of wave energy flux output from TAWAS. This plot shows the normalised wave energy flux of the torsional Alfvén wave as it propagates upwards through the central tube.

numerical calculations. However, as we will see, the reflective term can cause significant damping to the total wave energy flux.

Here we will demonstrate two separate points. Firstly, that the effect of reflection causes wave damping that is significantly different from the case where reflection is neglected entirely. To do this we will need to compare the solutions from TAWAS to those from an altered version of TAWAS that does not include wave reflection. We have named this altered version norefl and it can also be found at https: //github.com/calboo/TAWAS under the filename norefl.pro.

Secondly, that the effect of our correction to the formula caused wave damping that is significantly different from the uncorrected formula in which reflection is included. To do this will need to compare the solutions from TAWAS to those from an altered version of TAWAS that includes wave reflection but does not include our correction to the formula. We have named this altered version $u$ ucorr and it can also be found at https://github.com/calboo/TAWAS under the filename uncorr.pro.

To compare the wave energy flux outputs from these three scripts (TAWAS, norefl and uncorr) we consider plots of $\Pi(z)$ from each script for two different cases. For both cases we set the magnetic and density scale heights to $H=20 \mathrm{Mm}$ and $H_{\rho}=100 \mathrm{Mm}$ respectively and the viscosity to $v=5 \times 10^{7} \mathrm{~m}^{2} \mathrm{~s}^{-1}$, this will allow us to see the effects of both wave reflection and of viscous dissipation.

For the first case we set the wave period to $T=60 \mathrm{~s}$. A graph of $\Pi(z)$ is shown for this case in fig. 6 . For the second case we set the wave period to $T=120 \mathrm{~s}$ increasing the effect of wave reflection and reducing the effect of phase mixing. A graph of $\Pi(z)$ is shown for this case in fig. 7 .

We can see from fig. 6 that when $T=60 \mathrm{~s}$ the effect of wave reflection would seem large if the uncorrected formula is used, however, once the formula is corrected the solution including reflection is very similar to the solution without wave reflection. In fig. 7, on the other hand, we can see that when $T=120 \mathrm{~s}$ the effect of wave reflection is still significant even when the corrected formula is used.

From these graphs then, we can see that the effect of wave reflection should be included in our analysis as in some cases it can have a significant impact on wave damping. We can also see that our correction to the formula significantly changes the predicted amount

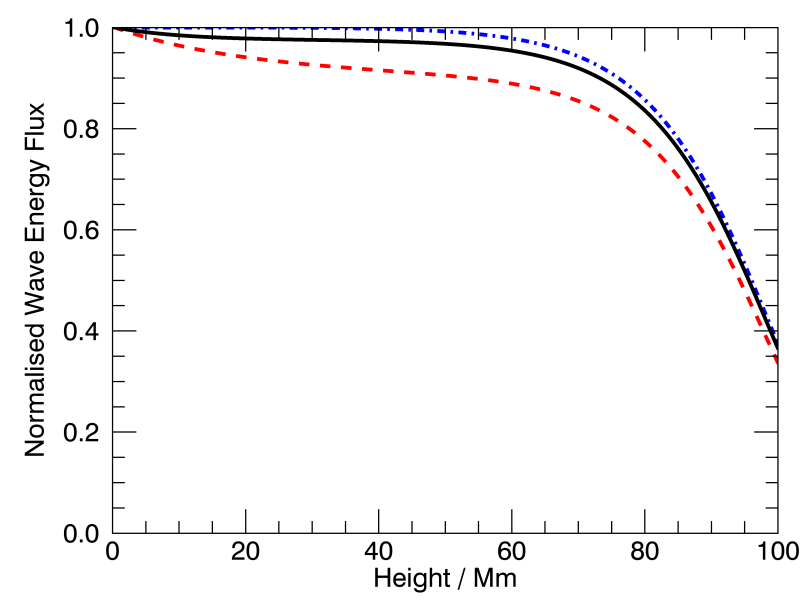

Figure 6. Graph of normalised wave energy flux $\Pi(z)$ for the case in which $H=20 \mathrm{Mm}, H_{\rho}=100 \mathrm{Mm}, v=5 \times 10^{7} \mathrm{~m}^{2} \mathrm{~s}^{-1}$ and $T=60 \mathrm{~s}$. The dot-dashed blue line show results from the formula in which reflection is neglected, the dashed red line shows results from the formula that includes reflection but does not include our correction and the black line shows results from our corrected formula.

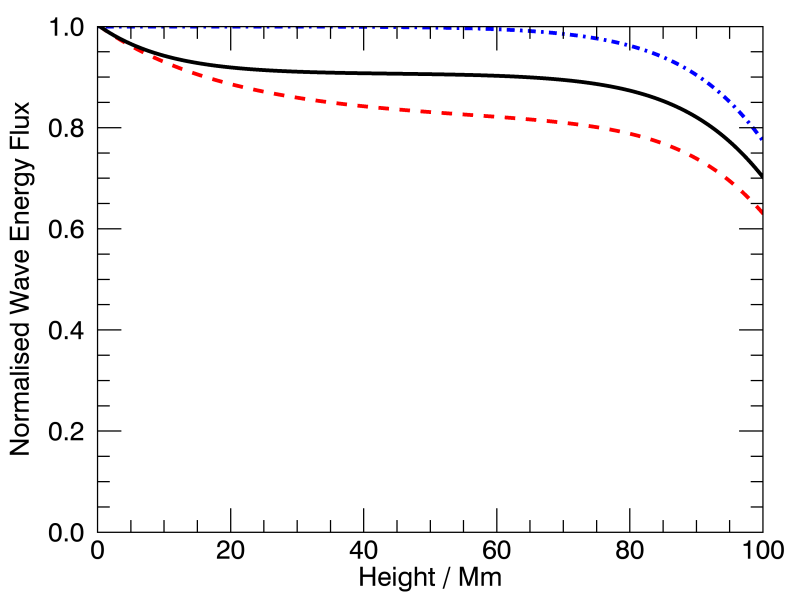

Figure 7. Graph of normalised wave energy flux $\Pi(z)$ for the case in which $H=20 \mathrm{Mm}, H_{\rho}=100 \mathrm{Mm}, v=5 \times 10^{7} \mathrm{~m}^{2} \mathrm{~s}^{-1}$ and $T=120 \mathrm{~s}$. The dot-dashed blue line show results from the formula in which reflection is neglected, the dashed red line shows results from the formula that includes reflection but does not include our correction and the black line shows results from our corrected formula.

of wave damping when compared to results from the uncorrected formula.

It is important when considering these graphs to note that, only the damping caused by phase mixing will heat up the corona through viscous dissipation. It is therefore incorrect to equate all of the damping of wave energy flux to viscous heating power. At first glance this might suggest that neglecting wave reflection is a sensible idea, however, note that in fig. 6 that the wave energy flux for both our corrected formula and for the solution with no reflection converge at 
higher heights. This suggests that if wave reflection is neglected then more power will be erroneously attributed to viscous heating.

These graphs not only demonstrate the significance of our correction to the formula they also demonstrate the importance of calculating damping due to wave reflection. Wave spectra of Alfvén waves in the corona suggest that partial reflection of the waves occurs and is a frequency dependent phenomena (Morton et al. 2015) as with our analytical solutions. By explicitly calculating the effect of wave reflection we can say with more certainty in which cases it can truly be neglected.

\section{ANALYTICAL PARAMETER STUDIES}

Using our corrected analytical formula and our numerical solver TAWAS we can now find solutions for a variety of different cases. We now conduct parameter studies to identify the cases in which damping of the torsional Alfvén wave is strongest.

This is necessary for two reasons. Firstly, we would like to know the conditions in which we expect enhanced phase mixing to dissipate a significant portion of the Alfvén waves energy through viscous dissipation. This will help to identify the conditions and regions of the solar corona in which phase mixing can fulfil the coronal heating requirement.

Secondly, we would like to test the validity of our analytic formula in the limit of strong Alfvén wave damping. When wave damping, in the form of either reflection or phase mixing, occurs over length scales comparable to the wavelength of the propagating wave then the WKB approximation is no longer valid, we say that these cases are beyond the WKB limit.

By identifying the cases for which wave damping is strongest we can later test the validity of our analytic formula beyond the WKB limit by comparing results from TAWAS to results from codes which solve the governing equations directly using finite differencing methods for these cases. Although we do not expect our formula to be accurate beyond the WKB limit, it is not clear whether we should expect more or less damping than predicted in these cases.

\subsection{Magnetic and Density Scale Height Parameter Study}

To begin with we perform a parameter study to investigate the effect of the magnetic and density scale heights $H$ and $H_{\rho}$. We run TAWAS for values of $H$ and $H_{\rho}$ between 20 and $100 \mathrm{Mm}$, viscosity and wave period are fixed at $v=5 \times 10^{7} \mathrm{~m}^{2} \mathrm{~s}^{-1}$ and $T=60 \mathrm{~s}$. We very broadly want to measure the total wave damping for each case, to quantify this we consider the normalised wave energy flux remaining at height $z_{\max }$, that is $\Pi\left(z_{\max }\right) / \Pi(0)$.

In fig. 8 we see a contour plot of $\Pi\left(z_{\max }\right) / \Pi(0)$ for the different values of $H$ and $H_{\rho}$ used in this study. We can see that for $v=5 \times 10^{7}$ $\mathrm{m}^{2} \mathrm{~s}^{-1}$ and $T=60 \mathrm{~s}$ there is only significant damping when the magnetic field lines are highly divergent. In fact the only cases where $\Pi\left(z_{\max }\right) / \Pi(0)<0.9$ were those with $H=20 \mathrm{Mm}$. Furthermore we can see that the damping increases as the density scale height is increased.

These results are what one would expect. The mechanism of enhanced phase mixing is greater for equilibria with highly divergent field lines and weak density stratification. The divergence of the magnetic field lines causes a decrease in Alfvén speed with height, this in turn decreases the wavelength of propagating waves leading to stronger transverse gradients and increased phase mixing. Conversely atmospheric stratification increases the Alfvén speed with height, increasing the wavelength of waves and reducing the effect of phase mixing.

By looking at graphs of $\Pi(z)$, such as the graph shown in fig. 5, for each individual case we can also see that wave reflection is strongest for low values of $H$ and high values of $H_{\rho}$. This is however for a different reason. Wave reflection is stronger when Alfvén waves encounter strong longitudinal gradients in the Alfvén speed. In the case of highly divergent magnetic field lines and weak density stratification, the gradient in Alfvén speed along field lines, i.e. in the direction of wave propagation, is greatest and hence the effect of wave reflection is strongest.

\subsection{Viscosity-Period Parameter Study}

Having identified the scale heights for which wave damping is the strongest we move on to investigate the effects of changing the viscosity, $v$, and the wave period, $T$. We conduct a second parameter study using TAWAS. We vary $v$ between $1 \times 10^{-4}$ and $v=5 \times 10^{7}$ $\mathrm{m}^{2} \mathrm{~s}^{-1}$ and $T$ between 15 and $120 \mathrm{~s}$. For this parameter study our scale heights are fixed at $H=20 \mathrm{Mm}$ and $H_{\rho}=100 \mathrm{Mm}$ as these values provide the strongest wave damping of all the values we have considered.

In fig. 9 we see a contour plot of $\Pi\left(z_{\max }\right) / \Pi(0)$ for the different values of $v$ and $T$ used in this study. This plot is particularly good at demonstrating the different dependencies of phase mixing and wave reflection. Phase mixing is stronger for shorter period waves and higher viscosities whilst wave reflection is stronger for longer period waves and is independent of viscosity.

We can see that for higher viscosities where viscous dissipation through phase mixing is the dominant cause of wave damping, the damping decreases as the period is increased because of the reduced effect of phase mixing. In contrast for low viscosities where there is very little wave damping caused by phase mixing, we can see that wave damping increases as the period is increased due to the growing effect of wave reflection.

We can also see that in the short period limit where phase mixing is strong and wave reflection is weaker there is a very strong dependence of the wave energy on viscosity. In the long period limit, however, the wave damping is mostly independent of the viscosity as it is caused primarily by wave reflection.

\section{FINITE DIFFERENCE SOLVER: WIGGLEWAVE}

Now that we have determined the parameter space for which wave damping is the strongest we can test the validity of the analytical solution both within and beyond the limits of the WKB approximation used in its derivation. We will do this by comparing the results for the Alfvén wave propagation calculated in TAWAS to the results from WiggleWave, a FORTRAN code written to solve the linearised governing equations for this system, eqs. (5) and (6), directly using finite differencing methods.

Wiggle Wave uses a fourth-order central difference scheme for calculating spatial derivatives and a fourth-order Runge-Kutta (RK4) scheme for updating at each timestep. WiggleWave calculates solutions for $v$ and $b$ over two-dimensional grid in radius $r$ and height $z$, the wave envelopes for $v$ and $b$ are also calculated. The source code and more details for WiggleWave can be found at https://github.com/calboo/Wigglewave.

For simulations in WiggleWave the grid resolution used over the domain was the same as in TAWAS, that is to $500 \times 2000$, in the $r$ and $z$ directions respectively. Although exponential damping regions 


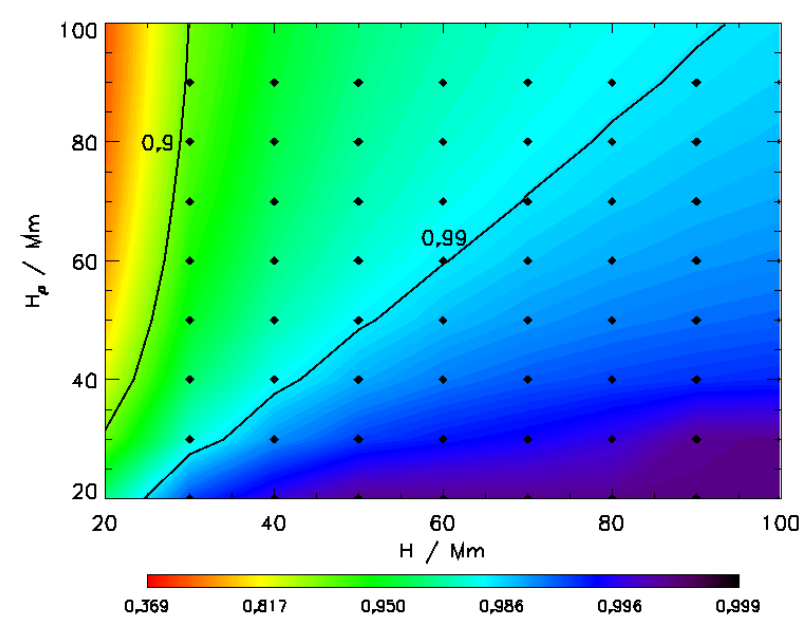

Figure 8. Contour plot showing values of the normalised wave energy flux, $\Pi\left(z_{\max }\right) / \Pi(0)$, from our scale height parameter study. Note that the colour scale is logarithmic. The magnetic scale height $H$ is on the $x$-axis and the density scale height $H_{\rho}$ is on the $y$-axis. The diamond symbols represent data points from the parameter study and the thick line contours indicate where $\Pi\left(z_{\max }\right) / \Pi(0)$ has values of 0.9 and 0.99 .

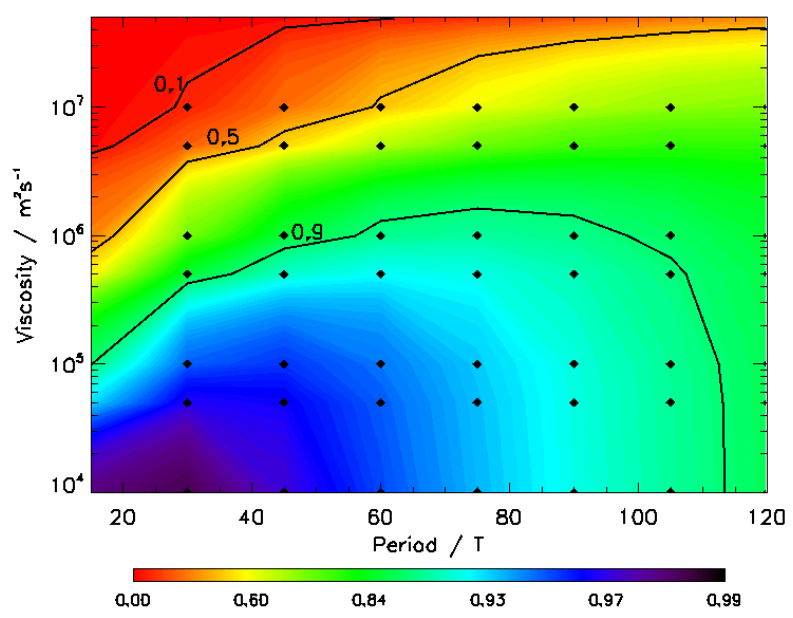

Figure 9. Contour plot showing values of the normalised wave energy flux, $\Pi\left(z_{\max }\right) / \Pi(0)$, from our viscosity-period parameter study. Note that the colour scale is logarithmic. The wave period $T$ is on the $x$-axis and the kinematic viscosity $v$ is on the $y$-axis. The diamond symbols represent data points from the parameter study and the thick line contours indicate where $\Pi\left(z_{\max }\right) / \Pi(0)$ has values of $0.1,0.5$ and 0.9 .

were also included for the upper vertical and outer radial boundaries when necessary, to prevent wave reflection from these boundaries. The numerical setup in WiggleWave used was identical to that used in TAWAS and described in section 2.3 except that wave driving at the lower boundary was prescribed within the tube boundary $r=r_{0}$ as,

$$
\begin{aligned}
& v=u_{0} \frac{r}{r_{0}}\left(1-\left(\frac{r}{r_{0}}\right)^{2}\right) \sin \left(\omega\left(\frac{z}{V_{A}}-t\right)\right), \\
& b=-u_{0} \frac{r}{r_{0}}\left(1-\left(\frac{r}{r_{0}}\right)^{2}\right) \sin \left(\omega\left(\frac{z}{V_{A}}-t\right)\right) \sqrt{\mu_{0} \rho}
\end{aligned}
$$

where we have approximated the lower boundary as a flat magnetic surface.

First, we compare the results of TAWAS and WiggleWave in a scenario where wave damping is relatively weak so that we can compare the results for cases in which the WKB approximation is valid. We set the magnetic scale height to $H=50 \mathrm{Mm}$ and a density scale height to $H_{\rho}=50 \mathrm{Mm}$, the kinematic viscosity is set to $v=5 \times 10^{7} \mathrm{~m}^{2} \mathrm{~s}^{-1}$. We consider cases with different wave periods, $T \in[10,30,60,90,120] \mathrm{s}$. Each case in this scenario was run for $3000 \mathrm{~s}$ to allow the wave to fully propagate throughout the domain and reach a state of quasi-equilibrium.

The data in figs. 10 and 11 is from the case where $T=10 \mathrm{~s}$. fig. 10 shows contour plots of the velocity perturbations from both TAWAS and WiggleWave as well as the difference between the envelopes of these two wave profiles. We can see that, ignoring the phase difference, the wave contours are almost identical. The maximum wave amplitude is about $60 \mathrm{~km} \mathrm{~s}^{-1}$ whilst the difference between the wave envelopes is well below $1 \mathrm{~km} \mathrm{~s}^{-1}$ over most of the domain. Similarly fig. 11 shows contour plots of the magnetic field perturbations from both TAWAS and WiggleWave as well as the difference between the envelopes of these two wave profiles. Again we can see that the wave contours are almost identical. The maximum wave amplitude is about $50 \mu T$ whilst the difference between the wave envelopes is below 0.5 $\mu T$ over most of the domain.

We can also compare graphs of the wave energy flux $\Pi(z)$ across magnetic surfaces of increasing height. fig. 12 shows graphs of the normalised wave energy flux against the height at which each magnetic surface intersects the $z$-axis for the case where $T=10 \mathrm{~s}$. We can see that although the curves from TAWAS and WiggleWave show significant damping at higher heights due to enhanced phase mixing, the difference between the two curves is negligible. In fact, the maximum difference in wave energy flux for this case is only $1.2 \%$. For cases with longer wave periods the damping is much less and the difference between the wave energy flux curves is always less than $1 \%$. This shows that within the limits of the WKB approximation the analytical model provides a valid solution to the linear governing equations.

Now we consider a different scenario to test the predictions of the analytical solution beyond the limits of the WKB approximation. We use a magnetic scale height of $H=20 \mathrm{Mm}$ and a density scale height of $H_{\rho}=100 \mathrm{Mm}$ to maximise wave damping. Again we set the kinematic viscosity to $v=5 \times 10^{7} \mathrm{~m}^{2} \mathrm{~s}^{-1}$ and consider cases with different wave periods, $T \in[10,30,60,90,120] \mathrm{s}$. Each case in this scenario was run for $4500 \mathrm{~s}$ to allow the wave to fully propagate throughout the domain and reach a state of quasi-equilibrium.

When comparing the wave envelopes for this scenario it is immediately obvious that there are differences between the TAWAS and WiggleWave solutions in all cases. This shows that indeed the WKB approximation used in the analytical solution is no longer valid when wave damping is strong, as we would expect. The question then is whether the wave damping and crucially the viscous damping, which is relevant to coronal heating, is stronger or weaker than the analytical model predicts.

To answer this question we can compare the graphs of the wave energy flux $\Pi(z)$ for these cases, fig. 13 shows a graph of the nor- 

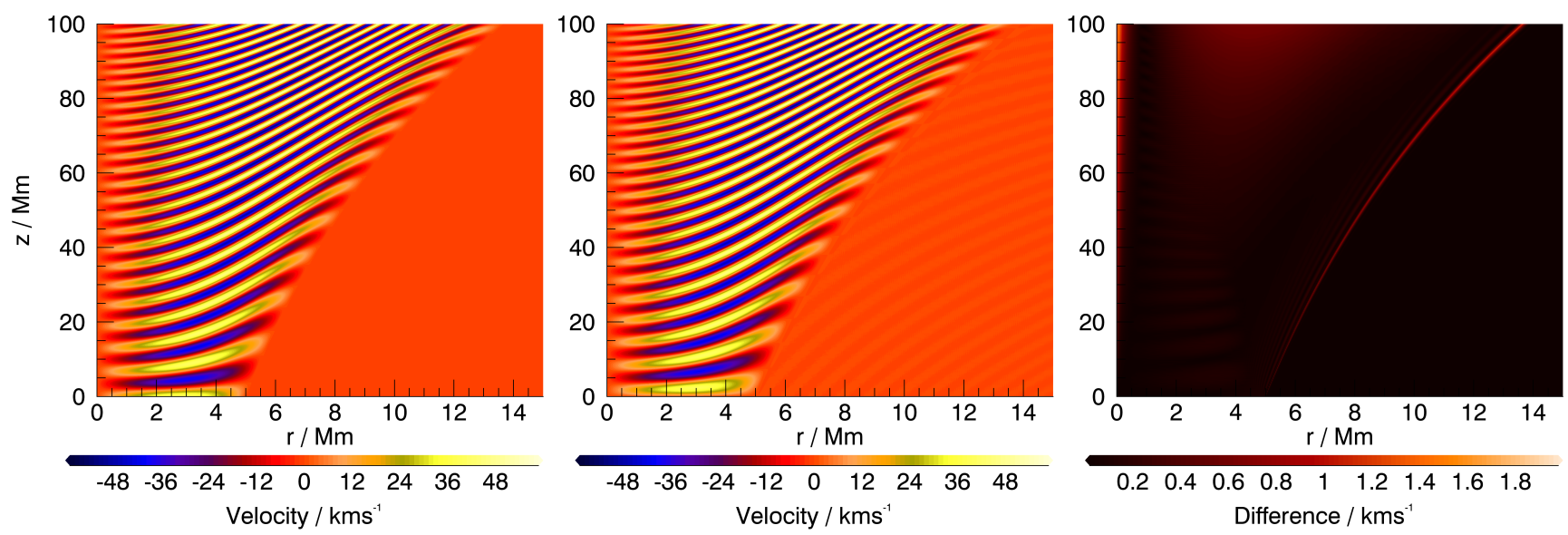

$\begin{array}{lllllllll}0.2 & 0.4 & 0.6 & 0.8 & 1 & 1.2 & 1.4 & 1.6 & 1.8\end{array}$

Difference / $\mathrm{kms}^{-1}$

Figure 10. Contour plots of the velocity profile for the torsional Alfvén wave, in the case where $T=10 \mathrm{~s}$, as calculated using TAWAS (left) and simulated using WiggleWave (centre) as well as a contour plot of the difference between the envelopes of these two wave profiles (right).
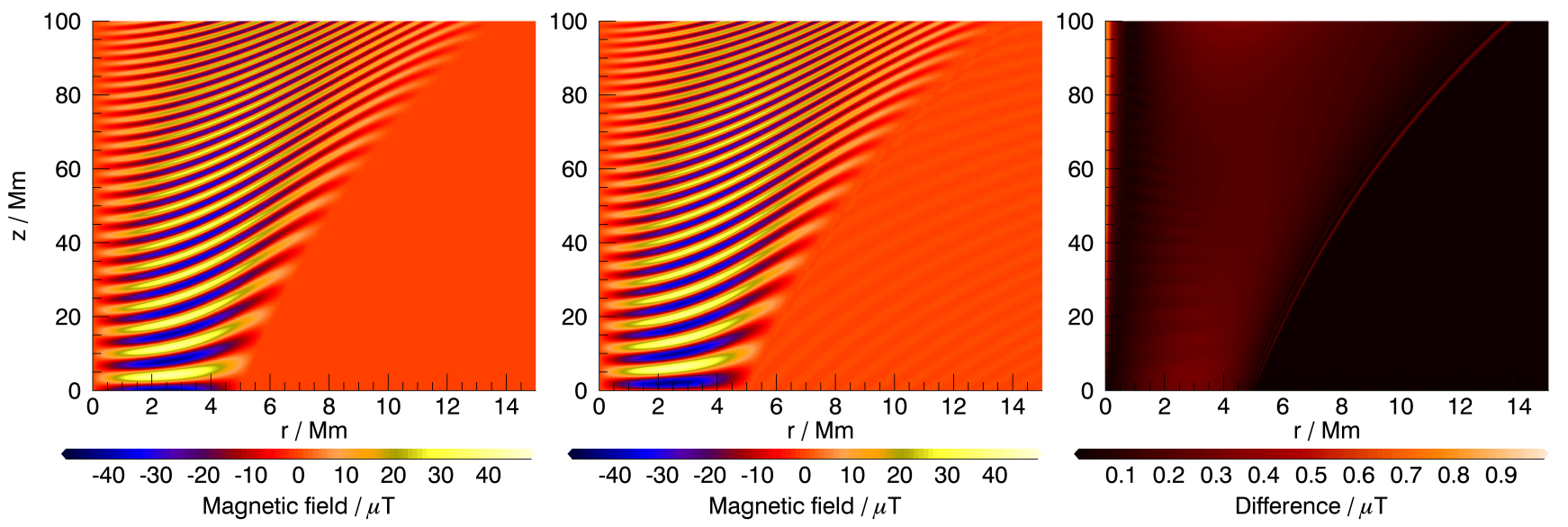

Figure 11. Contour plots of the magnetic field profile for the torsional Alfvén wave, in the case where $T=10 \mathrm{~s}$, as calculated using TAWAS (left) and simulated using WiggleWave (centre) as well as a contour plot of the difference between the envelopes of these two wave profiles (right).

malised wave energy flux for the case where $T=10 \mathrm{~s}$ and fig. 14 for the case where $T=60 \mathrm{~s}$. We can see in fig. 13 that in the case of $T=10 \mathrm{~s}$ the wave is fully damped before it reaches $100 \mathrm{Mm}$ for both solutions, we can also see that the WiggleWave solution shows stronger damping that the analytic solution from TAWAS. We can see in fig. 14 that in the case of $T=60 \mathrm{~s}$ the wave is strongly damped at $100 \mathrm{Mm}$ height but much more strongly in the WiggleWave solution than in the analytic solution from TAWAS. This demonstrates that beyond the WKB limit the analytic solution actually under-predicts the wave damping. As a side note, this graph also shows the effect of wave reflection in the lower domain for both solutions.

We can see in fig. 13 that for the case of $T=10 \mathrm{~s}$ the wave damping is caused almost entirely by viscous dissipation. Looking at fig. 14, however, we can see that for the case of $T=60 \mathrm{~s}$ the effect of wave reflection of wave energy flux is visible in the lower part of the domain for both the TAWAS and WiggleWave results. This is because the longer period wave has a larger wavelength and is therefore more prone to reflection, although as the wave propagates its wavelength decreases and so does the effect of wave reflection. It is interesting to note that solutions only diverge when viscous damping becomes strong due to enhanced phase mixing higher up in the domain.

\section{CONCLUSIONS}

In this paper we analytically and numerically studied the enhanced phase mixing of torsional Alfvén waves in an expanding magnetic flux tube embedded within a stratified coronal atmosphere. This type of structure is typical of a coronal plume or divergent coronal loop. We began in section 2 by formulating an analytical solution to the linearised governing equations eqs. (5) and (6) by following the derivation in (Ruderman \& Petrukhin 2018), that uses the WKB approximation, and making a correction toward the end of the formulation.

The IDL code TAWAS was presented in section 3. TAWAS calculates solutions using the analytic formula over a grid in radius, $r$ and height, $z$ as well as the total wave energy flux of the Alfvén wave over magnetic surfaces at different heights. In section 4 we showed that it is important to include the effect of reflection when considering Alfvén wave damping and that our correction to the analytical solu- 


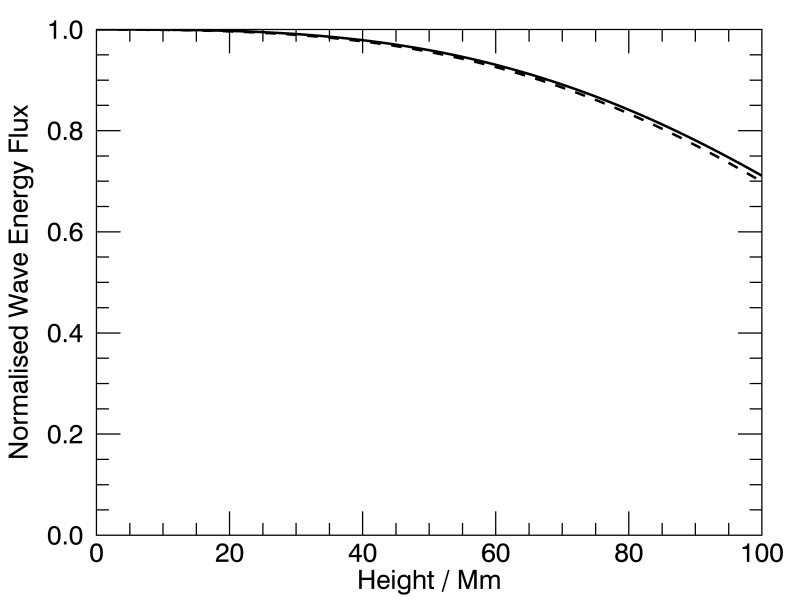

Figure 12. Graph of the normalised wave energy flux across magnetic surfaces plotted against the height at which each magnetic surface intersects the $z$-axis. This graph is for a scenario with $H=50 \mathrm{Mm}, H_{\rho}=50 \mathrm{Mm}, v=5 \times 10^{7}$ $\mathrm{m}^{2} \mathrm{~s}^{-1}$ and $T=10 \mathrm{~s}$. The wave energy flux from TAWAS is shown as a solid line and the wave energy flux calculated from the WiggleWave solution is shown as a dashed line.

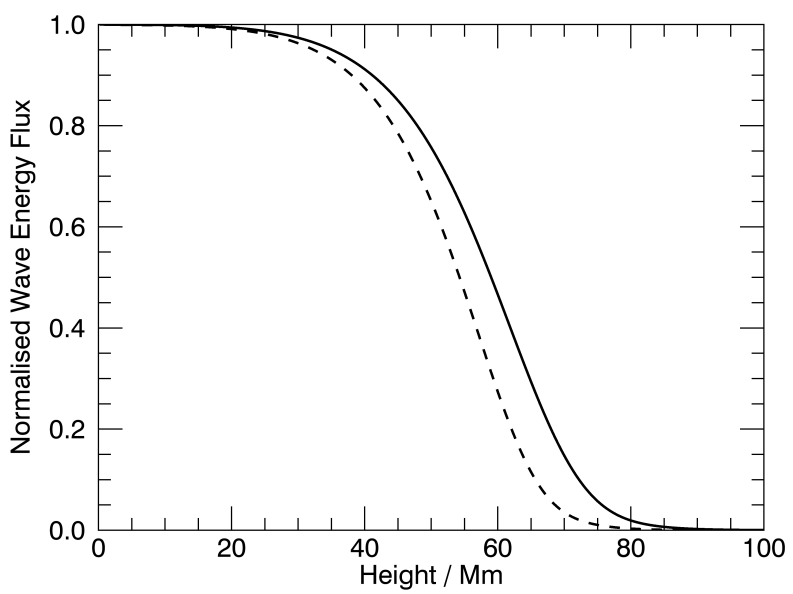

Figure 13. Graph of the normalised wave energy flux across magnetic surfaces plotted against the height at which each magnetic surface intersects the $z$-axis. This graph is for a scenario with $H=20 \mathrm{Mm}, H_{\rho}=100 \mathrm{Mm}, v=5 \times 10^{7}$ $\mathrm{m}^{2} \mathrm{~s}^{-1}$ and $T=10 \mathrm{~s}$. The wave energy flux from TAWAS is shown as a solid line and the wave energy flux calculated from the WiggleWave solution is shown as a dashed line.

tion decreases the amount of damping attributable to wave reflection relative to the uncorrected formula.

Then in section 5 we used TAWAS (https://github.com/ calboo/TAWAS) to perform two parameter studies to identify the parameter space in which Alfvén wave damping is significant over $100 \mathrm{Mm}$. In the first parameter study we showed that damping is only significant when the magnetic scale height is small $H<30 \mathrm{Mm}$ (i.e. for strongly divergent magnetic field lines) and the density stratification is $H_{\rho}$ is larger. In the second parameter study we showed that

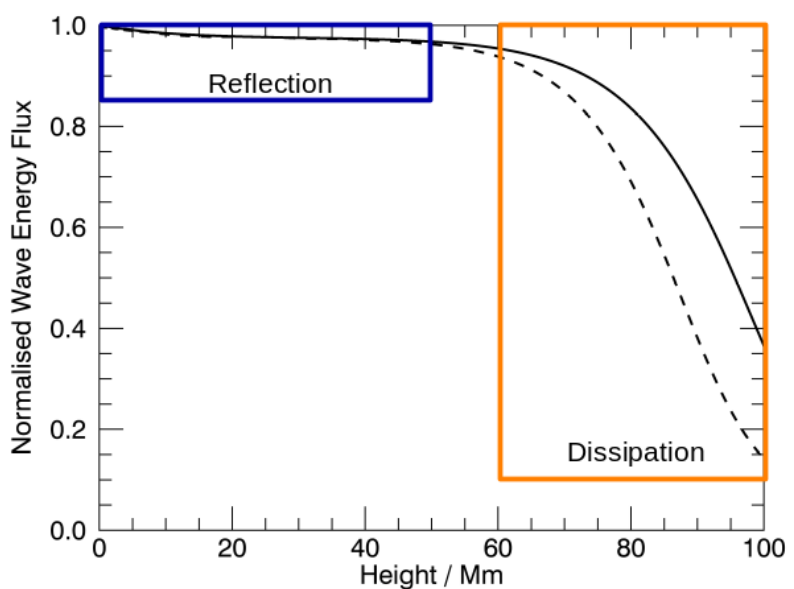

Figure 14. Graph of the normalised wave energy flux across magnetic surfaces plotted against the height at which each magnetic surface intersects the $z$-axis. This graph is for a scenario with $H=20 \mathrm{Mm}, H_{\rho}=100 \mathrm{Mm}, v=5 \times 10^{7}$ $\mathrm{m}^{2} \mathrm{~s}^{-1}$ and $T=60 \mathrm{~s}$. The wave energy flux from TAWAS is shown as a solid line and the wave energy flux calculated from the WiggleWave solution is shown as a dashed line. The annotated boxes shows regions where reflective damping (blue box) or viscous dissipation (orange box) are the dominant damping mechanism.

damping is stronger for lower period waves and higher kinematic viscosities.

To test the validity of our analytical solution, both within and beyond the limits of the WKB approximation, we wrote a finite difference solver in FORTRAN called Wigglewave (https: //github.com/calboo/Wigglewave) that directly solves the linearised governing equations eqs. (5) and (6) and is fourth-order accurate in time and space. In section 6 we compared results from TAWAS and Wigglewave. We showed that for scenarios with weak damping $\left(H=50 \mathrm{Mm}, H_{\rho}=50 \mathrm{Mm}, v=5 \times 10^{7}\right)$, the analytical solution matches the exact solution very well (to within $1 \%$ over most of the domain). Whereas for scenarios with strong damping ( $H=20 \mathrm{Mm}$, $H_{\rho}=100 \mathrm{Mm}$ ) the analytical solution under predicts wave damping compared to the exact solution calculated by Wigglewave. As a side note, our wigglewave results also showed the effect of wave reflection in graphs of the wave energy flux.

The simulation results from Wigglewave show that, assuming a reasonable transverse density gradient across the tube structure, the energy of torsional Alfvén waves can be fully dissipated within 100 $\mathrm{Mm}$, if the magnetic field lines are highly divergent, $H \sim 20 \mathrm{Mm}$, the wave period is short, $T \sim 10 \mathrm{~s}$ and a high value of anomalous kinematic viscosity, $v=5 \times 10^{7} \mathrm{~m}^{2} \mathrm{~s}^{-1}$, is assumed. These findings are similar to those in (Smith et al. 2007) which considers shear Alfvén waves and shows that shear waves of a similar amplitude are sufficient to satisfy the coronal heating requirement (Aschwanden 2004) when they are fully dissipated in sufficiently divergent active regions. Note, that in these simulations only monochromatic Alfvén wave driving has been considered, if instead a broad spectrum driver is considered as in (Tsiklauri \& Nakariakov 2001) and (Arber et al. 2016) then the heating requirement could potentially be fulfilled for lower values of viscosity.

Furthermore it is stated in (Smith et al. 2007) that additional physical effects, such as pressure, three-dimensionality and non-linearity, 
would lead to further heat deposition from the Alfvén waves. Indeed, in (Malara et al. 2000), it is shown that three dimensional effects can sometimes dissipate wave energy more efficiently than phase mixing, and in (Malara et al. 1996), it is shown that the interaction of Alfvén waves with a inhomogeneity can give rise to compressible perturbations. We will explore these effects further in Paper II using full MHD simulations. To summarise our findings in this paper:

(i) Wave reflection should be accounted for in the analytical solution for the enhanced phase mixing of torsional Alfvén waves. The Wigglewave results show that the effect of wave reflection is significant and can be accurately predicted by our corrected analytic formula.

(ii) The analytical solution for the enhanced phase mixing of torsional Alfvén waves is accurate at predicting the wave energy flux of the Alfvén wave within the limits of the WKB approximation but under-reports wave damping beyond the WKB limit.

(iii) The energy dissipated due to the enhanced phase mixing of torsional Alfvén waves is enough to fulfill the coronal heating requirement in a magnetic field with sufficiently divergent field lines, although this requires the application of anomalous viscosity and the presence of high frequency Alfvén waves.

\section{ACKNOWLEDGEMENTS}

C.B. would like to thank UK STFC DISCnet for financial support of his PhD studentship. This research utilized Queen Mary's Apocrita HPC facility, supported by QMUL Research-IT http://doi .org/ 10.5281 /zenodo .438045 .

\section{DATA AVAILABILITY}

All data used in this study was generated by either our analytical numerical solver TAWAS, https://gi thub.com/calboo/TAWAS, or our finite difference solver Wigglewave, https://github.com/ calboo/Wigglewave.

\section{REFERENCES}

Arber T. D., Brady C. S., Shelyag S., 2016, ApJ, 817, 94

Arregui I., 2015, Philosophical Transactions of the Royal Society of London Series A, 373, 20140261

Aschwanden M. J., 2004, Physics of the Solar Corona. An Introduction. Springer

Banerjee D., Teriaca L., Doyle J. G., Wilhelm K., 1998, A\&A, 339, 208

Braginskii S. I., 1965, Reviews of Plasma Physics, 1, 205

De Moortel I., Hood A., Arber T., 2000, Astronomy and Astrophysics, 354, 334

De Moortel I., Browning P., Bradshaw S. J., Pintér B., Kontar E. P., 2008, Astronomy and Geophysics, 49, 3.21

Doyle J. G., Teriaca L., Banerjee D., 1999, A\&A, 349, 956

Heyvaerts J., Priest E. R., 1983, A\&A, 117, 220

Liu J., Mcintosh S., Moortel I., Threlfall J., Bethge C., 2014, The Astrophysical Journal, 797

Malara F., Primavera L., Veltri P., 1996, ApJ, 459, 347

Malara F., Petkaki P., Veltri P., 2000, ApJ, 533, 523

Mathioudakis M., Jess D. B., Erdélyi R., 2013, Space Sci. Rev., 175, 1

Morton R. J., Tomczyk S., Pinto R., 2015, Nature Communications, 6, 7813

Parnell C. E., De Moortel I., 2012, Philosophical Transactions of the Royal Society of London Series A, 370, 3217
Petrukhin N. S., Ruderman M. S., Shurgalina E. G., 2018, Monthly Notices of the Royal Astronomical Society, 474, 2289

Ruderman M., 2021, Private Communucation

Ruderman M. S., Petrukhin N. S., 2017, A\&A, 600, A122

Ruderman M. S., Petrukhin N. S., 2018, A\&A, 620, A44

Ruderman M., Nakariakov V., Roberts B., 1998, Astronomy and Astrophysics, 338, 1118

Smith P. D., Tsiklauri D., Ruderman M. S., 2007, Astronomy \& Astrophysics, 475, 1111-1123

Soler R., Terradas J., Oliver R., Ballester J. L., 2017, ApJ, 840, 20

Soler R., Terradas J., Oliver R., Ballester J. L., 2021, ApJ, 909, 190

Stangalini M., Erdélyi R., Boocock C., Tsiklauri D., Nelson C. J., Del Moro D., Berrilli F., Korsós M. B., 2021, Nature Astronomy

Tsiklauri D., 2006, A\&A, 455, 1073

Tsiklauri D., 2009, Astronomy and Geophysics, 50, 5.32

Tsiklauri D., Nakariakov V. M., 2001, A\&A, 379, 1106 


\section{APPENDIX A: SOLUTION FOR A POTENTIAL AXISYMMETRIC MAGNETIC FIELD}

We require that the background magnetic field for our setup $\mathbf{B}_{\mathbf{0}}$ is a potential field, $\left(\nabla^{2} \mathbf{B}_{\mathbf{0}}=0\right)$, is axisymmetric, $\left(\partial_{\theta}=0\right)$, has no azimuthal component $\left(\mathbf{B}_{\mathbf{0}}=\left(B_{r}, 0, B_{z}\right)\right)$ and decays with height according to a characteristic scale height $H$.We begin by considering how the Laplace operator works in cylindrical coordinates for a random vector $\mathbf{A}$ and random function $f$,

$\nabla^{2} \mathbf{A}=\left(\begin{array}{c}\nabla^{2} A_{r}-\frac{A_{r}}{r^{2}}-\frac{2}{r^{2}} \frac{\partial A_{\theta}}{\partial \theta} \\ \nabla^{2} A_{\theta}-\frac{A_{\theta}}{r^{2}}+\frac{2}{r^{2}} \frac{\partial A_{r}}{\partial \theta} \\ \nabla^{2} A_{z}\end{array}\right)$

$\nabla^{2} f=\left(\frac{1}{r} \frac{\partial}{\partial r}\left(r \frac{\partial f}{\partial r}\right)+\frac{1}{r^{2}} \frac{\partial^{2} f}{\partial \theta^{2}}+\frac{\partial^{2} f}{\partial z^{2}}\right)$

Now substituting $\mathbf{B}_{\mathbf{0}}$ into our Laplace equation and applying our assumptions of axisymmetry and no azimuthal component we have equations for $B_{r}$ and $B_{z}$,

$\nabla^{2} \mathbf{B}_{\mathbf{0}}=\left(\begin{array}{c}\frac{1}{r} \frac{\partial}{\partial r}\left(r \frac{\partial B_{r}}{\partial r}\right)+\frac{\partial^{2} B_{r}}{\partial z^{2}}-\frac{B_{r}}{r^{2}} \\ 0 \\ \frac{1}{r} \frac{\partial}{\partial r}\left(r \frac{\partial B_{z}}{\partial r}\right)+\frac{\partial^{2} B_{z}}{\partial z^{2}}\end{array}\right)=\mathbf{0 .}$

\section{A1 Solution for $B_{r}$}

Let us first consider the solution for $B_{r}$,

$\frac{1}{r} \frac{\partial}{\partial r}\left(r \frac{\partial B_{r}}{\partial r}\right)+\frac{\partial^{2} B_{r}}{\partial z^{2}}-\frac{B_{r}}{r^{2}}=0$.

We try a separable solution $B_{r}=Z_{r}(z) R_{r}(r)$ which gives us,

$Z_{r} \frac{1}{r} \frac{\partial}{\partial r}\left(r \frac{\partial R_{r}}{\partial r}\right)+R_{r} \frac{\partial^{2} Z_{r}}{\partial z^{2}}-\frac{R_{r} Z_{r}}{r^{2}}=0$,

$Z_{r}\left(\frac{\partial^{2} R_{r}}{\partial r^{2}}+\frac{1}{r} \frac{\partial R_{r}}{\partial r}\right)+R_{r} \frac{\partial^{2} Z_{r}}{\partial z^{2}}-\frac{R_{r} Z_{r}}{r^{2}}=0$,

$\frac{1}{R_{r}}\left(\frac{\partial^{2} R_{r}}{\partial r^{2}}+\frac{1}{r} \frac{\partial R_{r}}{\partial r}\right)+\frac{1}{Z_{r}} \frac{\partial^{2} Z_{r}}{\partial z^{2}}-\frac{1}{r^{2}}=0$,

$\frac{1}{R_{r}}\left(\frac{\partial^{2} R_{r}}{\partial r^{2}}+\frac{1}{r} \frac{\partial R_{r}}{\partial r}\right)-\frac{1}{r^{2}}=-\frac{1}{Z_{r}} \frac{\partial^{2} Z_{r}}{\partial z^{2}}$.

As both sides are in separate variables they must each be equal to constant for this equality to hold. We call this constant $-k_{1}^{2}$, then we have,

$\frac{1}{R_{r}}\left(\frac{\partial^{2} R_{r}}{\partial r^{2}}+\frac{1}{r} \frac{\partial R_{r}}{\partial r}\right)-\frac{1}{r^{2}}=-k_{1}^{2}$,

$-\frac{1}{Z_{r}} \frac{\partial^{2} Z_{r}}{\partial z^{2}}=-k_{1}^{2}$
Note that we have used a $-k_{1}^{2}$ instead of $k_{1}^{2}$ so that we will end up with exponential solutions for $Z_{r}$ instead of oscillating solutions. The equation for $Z_{r}$ is then,

$\frac{\partial^{2} Z_{r}}{\partial z^{2}}=k_{1}^{2} Z_{r}$

The only solution for $Z_{r}$ that decays with characteristic scale height $H$ is,

$Z_{r}=e^{-z / H}$

This allows us to calculate $k_{1}$,

$\frac{\partial^{2} Z_{r}}{\partial z^{2}}=\frac{1}{H^{2}} Z_{r} \quad \Longrightarrow \quad k_{1}=\frac{1}{H}$

The equation for $R_{r}$ is,

$\frac{\partial^{2} R_{r}}{\partial r^{2}}+\frac{1}{r} \frac{\partial R_{r}}{\partial r}-\frac{1}{r^{2}}=-k_{1}^{2} R_{r}$

$\frac{\partial^{2} R_{r}}{\partial r^{2}}+\frac{1}{r} \frac{\partial R_{r}}{\partial r}+\left(k_{1}^{2}-\frac{1}{r^{2}}\right) R_{r}=0$,

now we define $p_{1}=k_{1} r$ and transform our equation so that it is in terms of $p_{1}$,

$\frac{\partial^{2} R_{r}}{\partial\left(p_{1}\right)^{2}}+\frac{1}{r} \frac{\partial R_{r}}{\partial p_{1}}+\left(1-\frac{1}{p_{1}^{2}}\right) R_{r}=0$,

we can write this as,

$\frac{\partial^{2} R_{r}}{\partial\left(p_{1}\right)^{2}}+\frac{1}{r} \frac{\partial R_{r}}{\partial p_{1}}+\left(1-\frac{m^{2}}{p_{1}^{2}}\right) R_{r}=0$,

where $\mathrm{m}=1$. The solution to this is a Bessel function of the first kind with $m=1$,

$R_{r}=J_{1}\left(p_{1}\right)=J_{1}\left(k_{1} r\right)=J_{1}\left(\frac{r}{H}\right)$.

The solution for $B_{r}$ is therefore,

$B_{r}=B_{0} e^{-z / H} J_{1}\left(\frac{r}{H}\right)$.

A2 Solution for $B_{z}$

Now we will consider the solution for $B_{z}$,

$\frac{1}{r} \frac{\partial}{\partial r}\left(r \frac{\partial B_{z}}{\partial r}\right)+\frac{\partial^{2} B_{z}}{\partial z^{2}}=0$.

We try a separable solution $B_{z}=Z_{z}(z) R_{z}(r)$ which gives us,

$Z_{z} \frac{1}{r} \frac{\partial}{\partial r}\left(r \frac{\partial R_{z}}{\partial r}\right)+R_{z} \frac{\partial^{2} Z_{z}}{\partial z^{2}}=0$

$Z_{z}\left(\frac{\partial^{2} R_{z}}{\partial r^{2}}+\frac{1}{r} \frac{\partial R_{z}}{\partial r}\right)+R_{z} \frac{\partial^{2} Z_{z}}{\partial z^{2}}=0$ 
$\frac{1}{R_{z}}\left(\frac{\partial^{2} R_{z}}{\partial r^{2}}+\frac{1}{r} \frac{\partial R_{z}}{\partial r}\right)+\frac{1}{Z_{z}} \frac{\partial^{2} Z_{z}}{\partial z^{2}}=0$,

$\frac{1}{R_{z}}\left(\frac{\partial^{2} R_{z}}{\partial r^{2}}+\frac{1}{r} \frac{\partial R_{z}}{\partial r}\right)=-\frac{1}{Z_{z}} \frac{\partial^{2} Z_{z}}{\partial z^{2}}$

As both sides are in separate variables they must each be equal to constant for this equality to hold. We call this constant $-k_{2}^{2}$, then we have,

$\frac{1}{R_{z}}\left(\frac{\partial^{2} R_{z}}{\partial r^{2}}+\frac{1}{r} \frac{\partial R_{z}}{\partial r}\right)=-k_{2}^{2}$

$-\frac{1}{Z_{z}} \frac{\partial^{2} Z_{z}}{\partial z^{2}}=-k_{2}^{2}$

Note that we have used a $-k_{2}^{2}$ instead of $k_{2}^{2}$ so that we will end up with exponential solutions for $Z_{z}$ instead of oscillating solutions. The equation for $Z_{z}$ is then,

$\frac{\partial^{2} Z_{z}}{\partial z^{2}}=k_{2}^{2} Z_{z}$

The only solution for $Z_{z}$ that decays with characteristic scale height $H$ is,

$Z_{z}=e^{-z / H}$.

This allows us to calculate $k_{2}$ which turns out to be the same as $k_{1}$,

$\frac{\partial^{2} Z_{z}}{\partial z^{2}}=\frac{1}{H^{2}} Z_{z} \quad \Longrightarrow \quad k_{2}=\frac{1}{H}$

The equation for $R_{z}$ is,

$\frac{\partial^{2} R_{z}}{\partial r^{2}}+\frac{1}{r} \frac{\partial R_{z}}{\partial r}=-k_{2}^{2} R_{z}$

$\frac{\partial^{2} R_{z}}{\partial r^{2}}+\frac{1}{r} \frac{\partial R_{z}}{\partial r}+k_{2}^{2} R_{z}=0$

now we define $p_{2}=k_{2} r$ and transform our equation so that it is in terms of $p_{2}$,

$\frac{\partial^{2} R_{z}}{\partial\left(p_{2}\right)^{2}}+\frac{1}{r} \frac{\partial R_{z}}{\partial p_{2}}+R_{z}=0$,

we can write this as,

$\frac{\partial^{2} R_{z}}{\partial\left(p_{2}\right)^{2}}+\frac{1}{r} \frac{\partial R_{z}}{\partial p_{2}}+\left(1-\frac{m^{2}}{p_{2}^{2}}\right) R_{z}=0$,

where $\mathrm{m}=0$. The solution to this is a Bessel function of the first kind with $m=0$,

$R_{z}=J_{0}\left(p_{2}\right)=J_{0}\left(k_{2} r\right)=J_{0}\left(\frac{r}{H}\right)$.

The solution for $B_{z}$ is therefore,

$B_{z}=B_{0} e^{-z / H} J_{0}\left(\frac{r}{H}\right)$

\section{A3 Solution for $B_{0}$}

The solution for the potential, axisymmetric magnetic field that decays with scale height $H$ is therefore,

$\mathbf{B}_{\mathbf{0}}=\left(\begin{array}{c}B_{0} e^{-z / H} J_{1}\left(\frac{r}{H}\right) \\ 0 \\ B_{0} e^{-z / H} J_{0}\left(\frac{r}{H}\right)\end{array}\right)$,

where $B_{0}$ is the characteristic magnetic field strength.

This paper has been typeset from a $\mathrm{T}_{\mathrm{E}} \mathrm{X} / \mathrm{LAT}_{\mathrm{E}} \mathrm{X}$ file prepared by the author. 